\title{
Development and Evaluation of Transit Signal Priority Strategies
}

\author{
Michael Garrow \\ Barton-AschmanAssociates, Inc. \\ Randy Machemehl \\ The University of Texas atAustin
}

\begin{abstract}
Research describing the effectiveness of providing signal priority to transit vehicles is presented. Results from previous studies indicate that the effectiveness of transit signal priority depends on a number offactors, including the type of transit route, the transit usage level, and the time of day. This research describes and evaluates several transit signal priority provision methods during both peak and off-peak times. Results indicate that providing signal priority during off-peak times is often justified, due to excess capacity available within the transportation network. However, during peak times, transit signal priority use is justified only when the transit usage level is high.
\end{abstract}

\section{Introduction}

In recent years, rising population levels coupled with low density development outside center cities has created a large increase in demand placed upon transportation networks in many urban areas. Building additional infrastructure to meet these increasing demand levels is expensive and time consuming and often encourages more travel. Therefore, in recent years, transportation professionals have shifted 
their efforts from building additional transportation infrastructure to operating the existing infrastructure more efficiently.

Encouraging travel in high occupancy vehicles, such as public transit, is one way to meet growing levels of travel demand while building little or no additional transportation capacity. However, low public transit usage plagues many urban areas, especially urban areas with low density development. In low density urban areas, travelers do not share many common origins or destinations, making it difficult for public transit to provide service levels competitive with the private automobile.

Therefore, policies providing priority to high occupancy vehicles have been initiated in many urban areas. Priority for public transit vehicles has been initiated in many urban areas over the past 25 to 30 years. Traditionally, priority for transit vehicles has been provided through signal timing advantages at signalized intersections. However, methods for providing signal priority for transit vehicles that consider the well-being of the transportation network as a whole should be developed.

This study examines work that previously has been performed in the transit signal priority arena, and proposes and evaluates methods of providing transit signal priority, during both peak and off-peak times.

Based on past transit signal priority studies, conditions favorable for transit signal priority success and strategies for its use are identified. These strategies were tested with both peak and off-peak traffic levels along a case study arterial network using computer simulation. Results from the computer simulation are evaluated over the entire arterial network to determine whether the various transit signal priority strategies provide an overall arterial network benefit.

\section{Literature Review}

Strategies for awarding priority for transit vehicles have been developed and tested in the field or using computer simulation over the past 20 to 30 years. Several signal priority algorithms are identified in a 1995 report by Sunkari of the Texas Transportation Institute (TTI) (Sunkari et al. 1995). These include both passive and active priority strategies. With passive priority, signalization plans are not affected by the presence or absence of transit vehicles. Shortening cycle lengths and splitting phases are examples of passive priority strategies. These algorithms are explained in 
greater detail in a related report by Urbanik (1977), also from TTI. Urbanik notes that shortening the cycle length along an arterial reduces stopped time delay to both transit vehicles, and private vehicles. However, the merits of a shortened cycle length must be weighed against the capacity reduction along the arterial.

Splitting phases refers to splitting transit's signal phase into multiple phases whose total time equals its original duration. This reduces the cycle length for the transit vehicle's approach, without altering the overall intersection cycle length.

Urbanik notes that active priority algorithms are different than passive priority algorithms, in that active priority measures are only taken in response to a transit vehicle signal priority request. Green extensions and red truncations are forms of active priority that steal green time from cross street approaches to be added to the end and beginning of the transit approach's green phase, respectively.

Active priority measures can be grouped into two categories: 1) unconditional priority, and 2) conditional priority. With unconditional priority (or preemption), a priority measure is granted whenever the transit vehicle calls for priority, subject to safety considerations. When using conditional priority, a transit vehicle is not necessarily given priority at an intersection every time priority is requested. Instead, the well-being of cross streets is considered before priority is granted to the transit vehicle's approach.

Bowen et al. (1994) describes how several parameters must be established before signal priority can effectively be granted to buses. The degree of intersection saturation below which priority may be granted is a highly important parameter. If this cutoff value is set too high, the usefulness of green extensions or red truncations will be lost when used in heavily congested environments. In addition, the intersection level of service may be further sacrificed through the excessive use of signal priority. However, if this cutoff value is set too low, buses that could have benefited from signal priority will not be granted a green extension or a red truncation. Green extension and red truncation lengths are also highly important variables. According to Bowen, the amount of priority should vary from intersection to intersection based on the amount of spare intersection capacity. 
Hounsell et al. (1996) tested active bus priority using simulation and determined that bus delay savings of 20-30 percent are possible without significant impacts to general traffic, when appropriate control settings (cutoff degree of saturation for priority use, etc.) are used with signal priority. Also, simulation showed increasing bus delay savings when signal priority was used with decreasing intersection saturation levels.

Hounsell also found that the use of green extensions alone, without red truncations, had the best overall impact upon traffic. The delay savings to transit are increased by supplementing green extensions with red truncations, but at a high cost to the general traffic.

In a separate paper, Hounsell and Wu (1995) identified additional issues involved with signal priority. The frequency of bus arrivals was identified as a key variable in determining appropriate signal priority strategies. In London, when buses were operated with one-minute headways, providing green extensions only was identified as the optimum strategy. When operating at headways shorter than one minute, adjusting signal timings to allow for bus progression was recommended.

A study conducted by the Municipality of Metropolitan Toronto (1991) found that streetcar delays and travel times decrease with the introduction of signal priority. Due to high transit usage in Toronto, this reduction in delay translates into substantial savings in passenger-minutes of delay. In addition, negative impacts to the cross streets resulting from priority were minimal. Further, green extensions were far more effective than red truncations. Often, red truncations were "lost" or could not be used by the streetcars along Queen Street.

Al-Sahili and Taylor used TRAF-Netsim to test the effectiveness of active signal priority (1995). Results indicate that arterial traffic suffered from overall increased delays whenever signal priority was initiated. Since the arterial traffic volumes were rather high, upon receiving signal priority, signal progression along the arterial was lost, resulting in increased downstream intersection delay. Therefore, along heavily traveled arterials, signal progression, rather than signal priority, appears to be of prime importance. 
The sensitivity of transit signal priority success to the ratio of arterial and cross street traffic volumes also was examined. The ratios of arterial traffic volumes to cross street traffic volumes selected for the analysis were 2:1, 3:1, and 5:1.

Results of the simulation indicate that negative impacts (in terms of increased delay per vehicle) introduced through the various signal priority techniques are significant at low volume ratios (2:1), but insignificant at high volume ratios (5:1). However, benefits from signal priority in terms of reduced bus travel times and delays decreases with increasing volume ratios because, at high volume ratios, signals are already timed to favor the bus approaches.

These past studies indicate the success of transit signal priority appears to depend on a number of factors, including the traffic characteristics at intersections where priority is used and the characteristics of the transit service itself. This study evaluates the effectiveness of signal priority and establishes guidelines for its use based on these traffic and transit service characteristics.

\section{Traffic Simulation Model-Based Data Collection}

The TRAF-Netsim simulation program was used as the analysis tool for this study. Since TRAF-Netsim is a link-node based micro-simulation model (USDOT 1998), it simulates all vehicles individually as they respond to traffic controls and other vehicles in the network.

TRAF-Netsim provided a useful tool for modeling transit signal priority. Passive transit signal priority was modeled using TRAF-Netsim by simply changing the signal timing characteristics in the simulation to reflect passive transit signal priority. TRAF-Netsim also proved to be very effective in modeling active signal priority, since it allows one to use multiple time periods within each simulation. Within each time period, one can alter input parameters such as signal timings, traffic volumes, lane channelizations, and turning percentages. With active signal priority, an intersection signal timing is temporarily altered to provide priority for a transit vehicle, then restored to its original timing upon the transit vehicle's departure. Active signal priority can be modeled within TRAF-Netsim by using the signal priority timing within a time period corresponding to the transit vehicle's arrival at the intersection. 
The intersection's original timing plan can be restored in the subsequent time period as the transit vehicle departs the intersection.

The graphical animation feature in TRAF-Netsim is also helpful when evaluating transit signal priority strategies. The animation feature displays a plan view of the network, where traffic movements and signal indications can be observed over the simulation duration. This graphical animation feature allows one to track the progress of buses through the network to determine where and when active signal priority is needed. Once a need for signal priority is identified, a time period can be inserted to initiate active signal priority.

Figure 1 shows the link-node representation of the Guadalupe-N. Lamar case study arterial in Austin, Texas, used for the transit signal priority analysis. Nodes 1 through 11 represent signalized intersections along the arterial, which extends roughly $4.1 \mathrm{~km}$ from the Koenig Lane and Lamar Boulevard intersection to the 27th Street and Guadalupe Street intersection. Buses share their right of way with private vehicles while operating along both northbound and southbound directions of the Guadalupe-N. Lamar arterial. Typically, two northbound and southbound lanes, as well as a continuous left turn lane, are present throughout the arterial.

\section{Off-Peak Time Period Transit Signal Priority}

Use of transit signal priority during the off-peak hours is promising because excess capacity available within the transportation network can be used for transit's advantage. In this study, the effectiveness of reduced signal cycle lengths and split phasing were examined in conjunction with local transit service, while unconditional priority was examined with respect to express transit service.

Using short cycle lengths as a passive transit signal priority strategy is appealing for several reasons. First, benefits to transit can be realized with little monetary cost. Implementing a policy of short cycle lengths along an arterial is a passive form of transit signal priority and is, therefore, in effect even in the absence of transit vehicles. Therefore, vehicle detection equipment is not necessary.

In addition, unlike most forms of active transit signal priority, a policy of short cycle lengths does not penalize vehicles along the cross streets by using a portion of their green time to favor transit vehicles. 


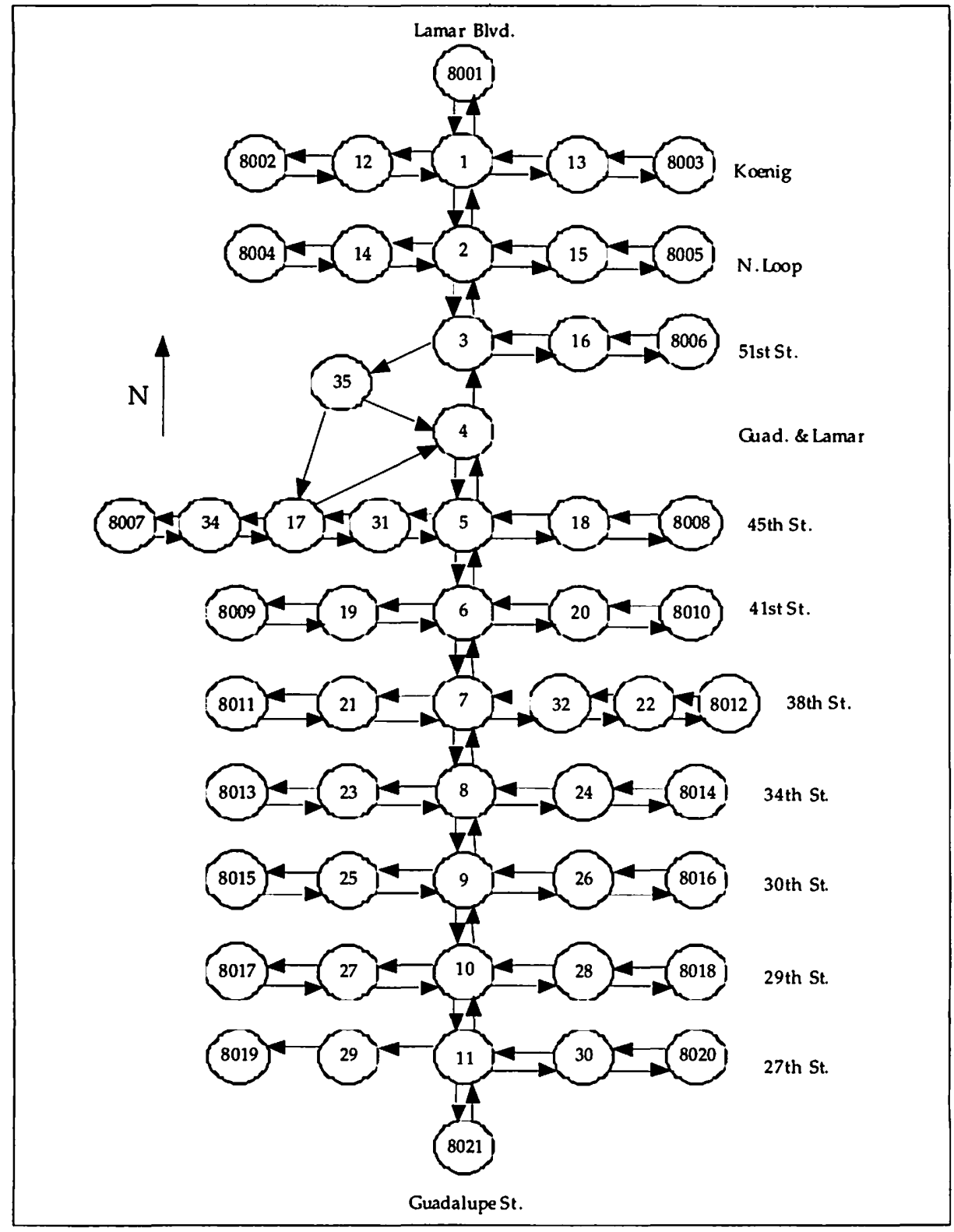

Figure 1. Link-node representation of Guadalupe-N. Lamar arterial. 
The effectiveness of granting passive signal priority to local transit vehicles with shortened cycle lengths was evaluated using TRAF-Netsim by comparing the performance of the Guadalupe-N. Lamar arterial under existing and passive priority signal control. Existing off-peak cycle lengths along the Guadalupe-N. Lamar arterial is 100 seconds. There is no evidence that shows that these 100 -second off-peak cycle lengths are optimal to process off-peak traffic volumes. Therefore, passive priority was implemented by reducing these cycle lengths to 70 seconds. Three replicate simulation runs lasting one hour each were performed.

Simulation results indicate that a 70 -second cycle length benefited buses by reducing their average travel time. Average bus travel times along the northbound route decreased from 797 seconds with the use of 100 -second cycles to 768 seconds with the use of 70-second cycles. Benefits to the Southbound bus route were even more impressive, as the average travel time dropped 11 percent, from 814 seconds to 725 seconds with 70-second cycle lengths.

Meanwhile, cumulative delay statistics along cross street and arterial links generated by TRAF-Netsim showed that the overall performance of these links improved with shorter cycle lengths. This analysis shows that both buses and private vehicles benefit when the cycle length along an arterial is pushed closer to its optimum value in response to lower off-peak traffic volumes.

\section{Split Phasing}

Split phasing was also examined using TRAF-Netsim by splitting bus phases at most intersections along the Guadalupe-N. Lamar case study arterial and comparing the resulting delays and bus travel times to those of the base case.

Splitting phases refers to splitting transit's signal phase into multiple phases whose total time equals its original duration. This reduces the cycle length for the transit vehicle's approach without altering the overall intersection cycle length. The concept of split phasing is illustrated in Figure 2. As shown in Figure 2, under normal phasing, if a bus arrives at the intersection on a red signal indication, it may have to wait the length of phases $B$ and $C$ before it receives a green indication with phase $A$. With split phasing, if a bus receives a red indication, it will only wait at most the length of phase $\mathrm{B}$ or phase $\mathrm{C}$ before receiving its green indication. 

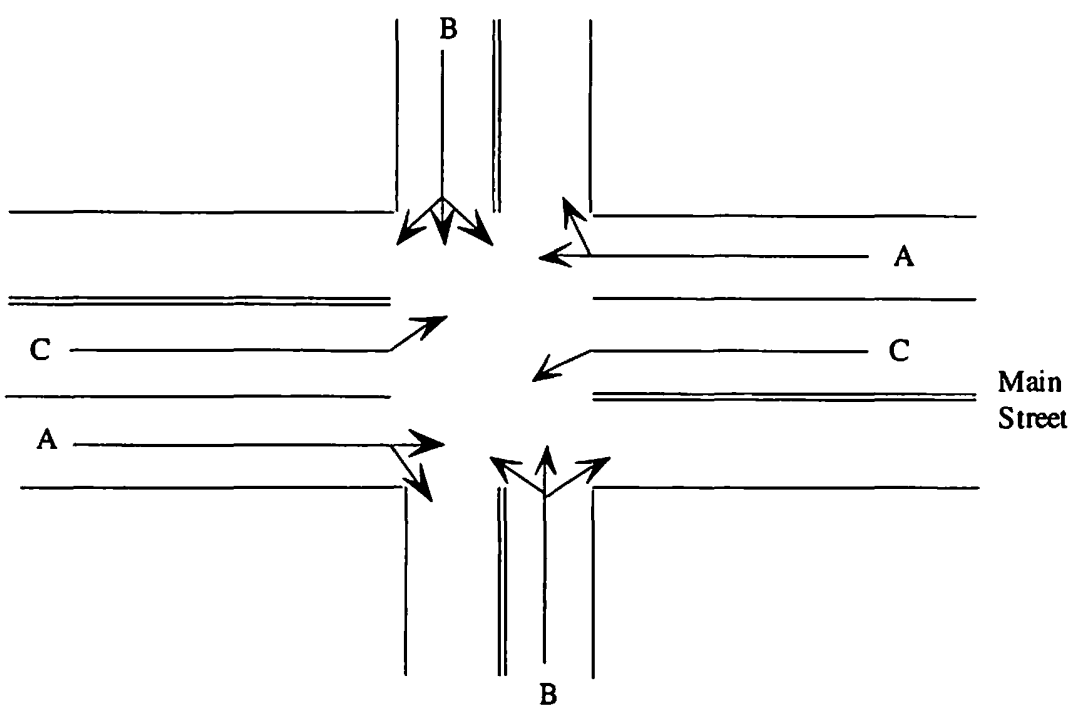

Cross

Street

Normal Phasing

A

B

C

Split Phasing

\begin{tabular}{|l|l|l|l|}
\hline A & B & A & C \\
\hline
\end{tabular}

Figure 2. Passive transit priority with split phasing.

Split phasing was evaluated using TRAF-Netsim and one-hour simulations. To account for variability and produce more robust results, three separate observations of the effects of split phasing and three base-case observations were performed using TRAF-Netsim. 
The impact of split phasing on local bus performance was mixed. The Northbound bus benefited from split phasing, as its average travel time dropped by nearly 10 percent, from 841 seconds to 757 seconds. However, the Southbound bus received no benefit as the average travel time increased slightly from 751 seconds to 767 seconds.

Meanwhile, split phasing had a minimal impact upon delay along both cross street and arterial links. Since split phasing does not impact the overall cross street or arterial green time, this result is not surprising.

\section{Unconditional Priority}

Unconditional priority is an active transit signal priority strategy where transit vehicles receive green extensions or red truncations whenever needed regardless of cross street queue lengths or the time since priority was last granted (Urbanik and Holder 1977).

While unconditional priority offers significant potential for transit, vehicles traveling on cross streets may feel severe negative impacts. Therefore, the use of unconditional priority should be reserved for express bus service during off-peak hours. Express bus service uses longer headways than local bus service, resulting in fewer priority calls over time, while off-peak traffic volumes enable cross streets to recover from each priority call more quickly than during the peak period.

The cross street degree of saturation (or saturation level) and the length of green extensions or red truncations made available to express buses are critical parameters to the success of unconditional priority.

The impact of unconditional priority on cross streets with various saturation levels was determined by performing several analyses using TRAF-Netsim. The first analysis examined how often unconditional priority would likely be triggered at the intersections along the Guadalupe-N. Lamar case study arterial. Unconditional priority was implemented along the northbound and southbound bus routes on the Guadalupe-N. Lamar arterial using the time period feature within TRAF-Netsim and the graphical animation. Three separate TRAF-Netsim simulations were performed in this manner for each direction. Therefore, a total of six buses (three northbound 
and three southbound) approached most intersections along the arterial within the analysis.

Results of this analysis indicated are shown in Table 1. As shown in Table 1, results indicate that unconditional priority will more likely be necessary at high volume intersections, such as 38th Street and 45 th Street intersections. Where the cross street volume is light, unconditional priority will rarely be triggered by the bus because the bus approach already receives a large fraction of intersection green time. Such is the case at the 51 st Street intersection.

The next analysis examined the effects of varying green extension and red truncation lengths and cross street saturation levels at several intersections.

The Eastbound approach of 38th Street, a cross street with heavy traffic, was examined first. Green extension or red truncation lengths available to the buses included 10 seconds of additional green, 20 seconds of additional green, and an unlimited amount of additional green as needed for the bus to traverse the intersection. In addition, the saturation level along the Eastbound approach of 38th Street varied from 0.62 (its existing saturation level) to 0.70 and 0.50 , respectively. To

\section{Table 1}

Percent of Time Priority Needed at Intersections

\begin{tabular}{lccc}
\hline \multicolumn{1}{c}{ Intersection } & $\begin{array}{c}\text { No. of Bus } \\
\text { Arrivals }\end{array}$ & $\begin{array}{c}\text { No. of } \\
\text { Priority Calls }\end{array}$ & $\begin{array}{c}\text { \% Priority } \\
\text { Needed }\end{array}$ \\
\hline $\begin{array}{l}\text { North Loop \& Lamar } \\
\text { (moderate volume cross street) }\end{array}$ & 6 & 2 & $33 \%$ \\
$\begin{array}{l}\text { 51st Street \& Lamar } \\
\text { (low volume cross street) }\end{array}$ & 6 & 1 & $17 \%$ \\
$\begin{array}{l}\text { 45th Street \& Guadalupe } \\
\text { (high volume cross street) }\end{array}$ & 6 & 3 & $50 \%$ \\
$\begin{array}{l}38 \text { th Street \& Guadalupe } \\
\text { (high volume cross street) }\end{array}$ & 6 & 6 & $100 \%$ \\
$\begin{array}{l}\text { 30th Street \& Guadalupe } \\
\text { (low volume cross street) }\end{array}$ & 6 & 2 & $33 \%$ \\
\hline
\end{tabular}


obtain robust results, three replicate simulations were conducted for each cell within this factorial experiment design.

Unconditional priority was simulated at the eastbound approach of 38th Street by taking green time from 38th Street in favor of the bus approach at 600 and 800 seconds into the simulation. Previous simulation results indicated that the Northbound bus typically requested priority at 38 th Street 600 seconds into the simulation, followed by a priority request from the Southbound bus 200 seconds later.

The second analysis examined the impact that unconditional priority had on the Westbound 45th Street approach, a cross street with only light to moderate off-peak hour traffic. This proceeded in a similar fashion to the last analysis (using three replicate runs per cell in the experiment), except saturation levels of $0.25,0.38$ (existing saturation level), and 0.50 were simulated. Also, because this is not a high volume intersection, simulation revealed that only 1 signal priority call would likely be necessary.

Results indicate that, placing a 10-second limit on the added green time, which the bus approach receives from the cross street, limits the impacts to the eastbound approach of 38th Street. Placing a 20-second limit on each signal priority call resulted in significant delay increases along the cross street, as delay levels hover in the range of 45 seconds per vehicle for roughly 500 seconds.

Similar results were encountered when the Eastbound 38th Street approach saturation level was lowered to 0.62 and 0.50 , when a 10 -second limit on the signal priority time protected the cross street from significant delay increases.

When unconditional priority was simulated along the Westbound 45th Street approach with a saturation level of 0.50 , unlimited priority increased the cross street delay producing effects lasting about 5 minutes. Both 10 -second and 20-second priority calls, however, had little impact upon delay. Similar results were encountered when the saturation level along the Westbound 45th Street approach was lowered to 0.38 .

However, when the cross street saturation level was reduced to 0.25 , enough excess capacity was available along the Westbound 45th Street approach to enable it to recover from even an unlimited priority call. Although the cross street delay 
increase resulting from an unlimited priority call (taking 30 to 40 seconds of green away from the cross street) was greater than when limits were imposed on signal priority, the cross street was not severely impacted.

Therefore, when the cross street intersection saturation levels drop below 0.25 , one might consider using unlimited signal priority because present signal timing should already heavily favor the bus approach. Therefore, the bus will rarely need to request priority, and the priority time needed will typically be fairly small.

The results of the analysis of unconditional priority at the Eastbound 38th Street approach and the Westbound 45th Street approach are summarized in Table 2.

\begin{tabular}{|lc|}
\hline \multicolumn{1}{|c|}{$\begin{array}{c}\text { Table } 2 \\
\text { Guidelines for Use of } \\
\text { Unconditional Priority } \\
\text { During Off-Peak Hours }\end{array}$} \\
\hline $\begin{array}{l}\text { Cross Street } \\
\text { Saturation Level }\end{array}$ & $\begin{array}{c}\text { Recommended Green } \\
\text { Extension or Red } \\
\text { Truncation Length }\end{array}$ \\
\hline Below 0.25 & Unbounded \\
0.25 to 0.35 & 20 Seconds \\
0.35 to 0.70 & 10 Seconds \\
\hline
\end{tabular}

\section{Peak Time Period Transit Signal Priority}

Implementation of transit signal priority during peak time periods is more difficult than during off-peak time periods. Because both cross streets and arterials are likely to be operating at higher degrees of saturation than during off-peak times, less excess transportation network capacity is available.

\section{Impact of Transit Signal Priority on Cross Street Delay}

To observe the effects of transit signal priority on cross street delay during the peak time period, the delay calculated within TRAF-Netsim at several cross streets was monitored as green time was taken from these approaches and given to the bus approach in the form of a green extension. 
Cross street saturation levels and green time taken from the cross street and provided to the bus approach were varied over the simulation runs. The resulting impacts were observed at two cross streets-the eastbound 38th Street approach and the westbound 45th Street approach.

The total simulation time used for every run was one hour and three replicate simulations were conducted for each cell within the factorial experiment. Within each run, a transit signal priority cycle was inserted in place of the normal signal timing at the particular intersection under examination once every 10 minutes. This mimics the arrival of a bus requesting priority once every 10 minutes (the assumed bus headway).

Results indicate that when 10-second green extensions were used in conjunction with a cross street saturation level of 0.8 , signal priority did not result in substantial increases in delay per vehicle along the cross street approaches.

When the cross street saturation level was raised to 0.9 in conjunction with the use of 10-second green extensions, the cross streets began to feel more substantial delay increases, causing the use of priority to become questionable. Increases in delay felt by cross streets did not readily dissipate with time.

When cross street saturation levels were raised to 1.0 and 10 -second green extensions were used, simulation results at both approaches revealed increasing delay levels over time with each signal priority initiation. This indicates that green time should not be taken from cross streets operating at saturation levels of 1.0 to award priority to transit vehicles along an arterial.

When the green extensions awarded to the bus approach were increased from 10 seconds to 20 seconds per priority call, larger delay increases were encountered along the cross streets.

However, when the cross streets were operating at saturation levels of 0.8 , enough excess capacity was available to allow them to recover from the impacts of the priority signal timing within two to three signal cycles following each priority call. However, it is unclear whether the benefits to transit from signal priority outweigh the increases in delay incurred by vehicles along the cross street. A more comprehensive analysis is required. 
As the saturation level of the Eastbound approach at 38th Street is increased to 0.9 , substantial increases in cross street delay relative to the base case were felt over the majority of these simulations, indicating that signal priority should not be used in this context.

The results of this analysis are summarized in Table 3. Under conditions where only minimal negative impacts are felt by cross streets due to signal priority, the use of signal priority may be appropriate. Should transit signal priority be used under conditions which generate moderate negative impacts along the cross streets, the resulting increases in delay along the cross streets should be closely scrutinized, while transit signal priority should be avoided under conditions that generate significant negative impacts to the cross streets.

\begin{tabular}{|lcc|}
\hline \multicolumn{3}{|c|}{$\begin{array}{c}\text { Table } 3 \\
\text { Negative Impacts Accruing on Cross Streets Due to } \\
\text { Signal Priority (Assumed Bus Headway }=10 \text { Minutes) }\end{array}$} \\
\hline $\begin{array}{l}\text { Cross Street } \\
\text { Saturation }\end{array}$ & $\begin{array}{c}\text { Green Extension } \\
=10 \text { Sec. }\end{array}$ & $\begin{array}{c}\text { Green Extension } \\
=20 \text { Sec. }\end{array}$ \\
\hline \begin{tabular}{lcc} 
Saturation Level $=0.8$ & Minimal & Moderate \\
Saturation Level $=0.9$ & Moderate & Significant \\
Saturation Level $=1.0$ & Significant & Significant \\
\hline $\begin{array}{l}\text { Minimal Impacts-Signal priority appropriate. } \\
\text { Moderate Impacts-Signal priority should be used with } \\
\text { caution; delays on side streets should be closely monitored. }\end{array}$ \\
Significant Impacts-Signal priority should be avoided.
\end{tabular} \\
\hline
\end{tabular}

\section{Arterial Street Impacts Upon Signal Priority Effectiveness}

This analysis examined how different characteristics of the bus arterial affect the success of active transit signal priority. In particular, two characteristics were examined: 1) the location of bus stops along the arterial (near-side versus far-side) and 2) the saturation level of the bus approach. 
TRAF-Netsim was used to examine the success of a green extension in allowing buses to traverse a test intersection without stopping in a variety of traffic conditions. In particular, the bus stop location, bus approach saturation level, and green extension length were all varied within simulation runs. For each scenario examined within the factorial experiment, 8 to 10 replicate observations were made using TRAF-Netsim.

Green extensions were triggered with the aid of TRAF-Netsim's graphical interface. Upon approaching the intersection, the graphics display would indicate whether the bus was in need of signal priority. If so, a time period would be inserted within the simulation that contained the signal priority signal timing. The outcome of this signal priority timing was then observed using TRAF-Netsim's graphical animation. Bus dwell times at the bus stop were simulated within TRAF-Netsim based on a dwell time distribution modeled within the simulator. This dwell time distribution was based on field data collected along the Guadalupe-N. Lamar corridor in Austin.

Tables 4 and 5 summarize the results of this analysis for near-side and far-side bus stops, respectively. For each scenario, a success rate for the green extension used is given. Success in this context indicates that the green extension enabled the bus to

\begin{tabular}{|lcccc|}
\hline \multicolumn{5}{c|}{ Table 4} \\
Success & Rate of Green Extensions (Near-Side Bus Stop) \\
\hline $\begin{array}{c}\text { Green } \\
\begin{array}{c}\text { Extension } \\
\text { Length }\end{array}\end{array}$ & $\begin{array}{c}\text { Bus Approach } \\
\text { Saturation } \\
\text { Level }\end{array}$ & $\begin{array}{c}\text { No. of } \\
\text { Attempted } \\
\text { Extensions }\end{array}$ & $\begin{array}{c}\text { No. of } \\
\text { Successful } \\
\text { Extensions }\end{array}$ & $\begin{array}{c}\text { Success } \\
\text { Rate }\end{array}$ \\
\hline 10 Seconds & Saturation $=0.8$ & 10 & 2 & $20 \%$ \\
10 Seconds & Saturation $=0.9$ & 10 & 1 & $10 \%$ \\
10 Seconds & Saturation $=1.0$ & 10 & 0 & $0 \%$ \\
20 Seconds & Saturation $=0.8$ & 10 & 3 & $30 \%$ \\
20 Seconds & Saturation $=0.9$ & 10 & 3 & $30 \%$ \\
20 Seconds & Saturation $=1.0$ & 10 & 0 & $0 \%$ \\
\hline
\end{tabular}




\begin{tabular}{|lcccc|}
\hline \multicolumn{5}{c|}{ Table 5 } \\
Success Rate of Green Extensions (Far-Side Bus Stop) \\
\hline $\begin{array}{c}\text { Green } \\
\begin{array}{c}\text { Extension } \\
\text { Length }\end{array}\end{array}$ & $\begin{array}{c}\text { Bus Approach } \\
\text { Saturation } \\
\text { Level }\end{array}$ & $\begin{array}{c}\text { No. of } \\
\text { Attempted } \\
\text { Extensions }\end{array}$ & $\begin{array}{c}\text { No. of } \\
\text { Successful } \\
\text { Extensions }\end{array}$ & $\begin{array}{c}\text { Success } \\
\text { Rate }\end{array}$ \\
\hline 10 Seconds & Saturation $=0.8$ & 8 & 5 & $63 \%$ \\
10 Seconds & Saturation $=0.9$ & 9 & 6 & $67 \%$ \\
10 Seconds & Saturation $=1.0$ & 10 & 5 & $50 \%$ \\
20 Seconds & Saturation $=0.8$ & 8 & 7 & $88 \%$ \\
20 Seconds & Saturation $=0.9$ & 9 & 8 & $89 \%$ \\
20 Seconds & Saturation $=1.0$ & 10 & 6 & $60 \%$ \\
\hline
\end{tabular}

avoid a red signal indication at the intersection, which it would have otherwise received.

As can be seen in Table 4, the presence of a near-side bus stop greatly hinders the effectiveness of green extensions. Near-side bus stops limit the success of green extensions because a significant portion, if not all, the green extension is wasted while passengers board and deboard at the near-side bus stop.

Table 5 shows the success rate of green extensions when used with a far-side bus stop configuration. Transit signal priority is much more successful when used with far-side bus stops, rather than near-side bus stops. With far-side bus stop configurations, the success of signal priority is no longer a function of the bus dwell time.

Table 5 further shows that the success rates of both the 10 - and 20 -second green extensions remain relatively constant as the bus approach degree of saturation increases from 0.8 to 0.9 . In particular, the success rate of the 20 -second green extensions along bus approaches with saturation levels of 0.8 or 0.9 looks extremely promising from the bus's viewpoint. However, as shown in the previous analysis, the use of 20-second green extensions causes moderate to significant increases in delay along cross streets operating at saturation levels above 0.8 . 
These findings identify the direct conflict arising between the success of signal priority and the negative impacts along cross streets resulting from signal priority. To determine whether the use of signal priority is justified, a comprehensive analysis that examines the overall net effect of signal priority upon an intersection is needed.

\section{Effectiveness of Signal Priority at an Isolated Intersection}

This analysis addresses the questions raised previously regarding whether signal priority can be justified by evaluating the overall net impact of transit signal priority at a single intersection.

The intersection of 38th Street and Guadalupe Street was simulated using TRAFNetsim. This intersection was chosen because the cross street volume along 38th Street is significant and, therefore, a definite conflict exists between arterial traffic along Guadalupe Street, which stands to benefit from signal priority, and the cross street traffic, which is negatively influenced by signal priority.

Based on the results of the last analysis, a far-side bus stop configuration is assumed. Also, a 10-minute bus headway is assumed and transit signal priority is assumed to only be used by buses traveling in the peak period direction (the northbound intersection approach).

The criteria that is used to address the effectiveness of transit signal priority at a single intersection is the travel time per person over all individuals approaching the intersection over a given time frame.

The time frame used for this analysis began 600 seconds into each TRAFNetsim simulation and ends 10 minutes later, at 1200 seconds. A green extension was used at the intersection 600 seconds into the simulation, and the effects of this green extension were examined over the following 10 minutes.

The analysis of signal priority effects at a single intersection was broken into the following three components: 1) the analysis of signal priority effects on travel time per person along non-bus approaches, 2) the analysis of signal priority effects on travel time per person for non-bus traffic along the bus approach, and 3) the analysis of signal priority effects on travel time per person on-board the bus.

The effects of signal priority along the non-bus approaches were monitored by acquiring cumulative travel time and vehicle counts along the three non-bus links 
600 and 1200 seconds into each simulation. These data were collected in conjunction with both 10 -second and 20-second green extensions, with each simulation accompanied by a base case where no signal priority was used. Three replicate runs were performed for each scenario described above.

Whereas the volumes along the non-bus approaches were fixed at the volumes consistent with peak period volumes from the Guadalupe-N. Lamar case study network, the bus approach volume was varied to create approach saturation levels of 0.8 , 0.9 , and 1.0. The effect of signal priority on non-bus traffic along the bus approach was also monitored by acquiring cumulative travel time and vehicle counts for nonbus traffic along the bus approach at 600 and 1200 seconds into each simulation. Three replicate runs were performed for each scenario described above.

Finally, data concerning the effect that signal priority had on the bus travel time along its approach were obtained by collecting the bus travel times from TRAFNetsim, using three replicate runs for each scenario tested.

Having collected statistics regarding the total travel times and number of vehicles processed along all 4 approaches of the 38th Street and Guadalupe Street intersection, the overall travel time per person at the intersection was calculated by assuming occupancy rates for the bus and the automobiles.

Upon assuming an auto occupancy of 1.2 and a bus occupancy of 25 , the measures of travel time per person shown in Table 6 were obtained.

As can be seen in Table 6, signal priority does not appear to significantly impact the overall travel time per person at the intersection. This result stems from the small bus share of roughly 2.9 percent of the total person-trips at the intersection. As a result, reducing the travel time for this small fraction of users had a negligible overall impact upon the travel time per person over the entire intersection.

In addition, when looking at Table 6 , one notices the superior performance of the 10-second green extension over the 20 -second green extension across all bus saturation levels. This finding indicates that a signal should accommodate its users. Larger green extensions place the signal timing of an intersection farther away from the original timing intended for individuals in autos, the intersection's major group 


\section{Table 6}

Travel Time per Person (Seconds/Person) at 38th Street and Guadalupe (Auto Occupancy = 1.2, Bus Occupancy $=25$ )

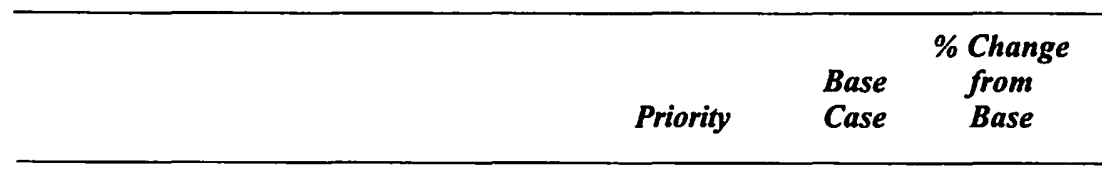

\section{0-Second Green Extension}

Bus approach saturation level $=0.8$

47.5

47.6

$-0.2$

Bus approach saturation level $=0.9$

49.1

49.9

$-1.7$

Bus approach saturation level $=1.0$

53.0

55.0

\section{0-Second Green Extension}

Bus approach saturation level $=0.8$

49.8

Bus approach saturation level $=0.9$

Bus approach saturation level $=1.0$

of users in this analysis. Similar results were encountered even when a bus occupancy of 50 passengers was assumed.

It is important to note that the findings from this analysis are based on 10minute headways and heavy automobile volumes taken from traffic counts at the 38th Street and Guadalupe Street intersection. Had the transit mode share been higher, the effectiveness of signal priority would likely increase.

\section{Effectiveness of Signal Priority within an Arterial Street Network}

The previous analysis showed transit signal priority to be largely ineffective at an isolated intersection with high cross street traffic volumes. However, in reality, transit signal priority systems are not operated only at isolated intersections, but along transit corridors, such as the Guadalupe-N. Lamar arterial. Only by looking at the overall impact of transit signal priority upon the entire arterial and its cross 
streets can one clearly determine whether the use of transit signal priority can be justified along the corridor.

The users of the Guadalupe-N. Lamar arterial network can be broken into three categories.

1) non-bus users traveling along the cross streets,

2) non-bus users traveling along the arterial, and

3) bus users traveling along the arterial.

Previous analyses have clearly indicated that non-bus users traveling along the cross streets suffer increased delays with the use of transit signal priority.

However, the effect that transit signal priority has upon non-bus users traveling along the arterial remains somewhat unclear. This group of users stands to benefit from the effects of transit signal priority as their overall green time increases with the use of transit signal priority. However, signal coordination along the arterial is also very important to the success of this user group. It is still unclear how transit signal priority affects signal coordination along an arterial.

Finally, bus users should realize improvements in service levels with the use of transit signal priority. However, as shown in the previous section, the benefits to this small group of users might not justify the negative impacts to cross street users or potential negative impacts to automobiles traveling along the arterial.

To determine the overall effect of transit signal priority upon the Guadalupe-N. Lamar arterial, this analysis quantifies the effects that transit signal priority has on the three user groups.

The transit signal priority implementation strategies which will be tested within this analysis are as follows:

Case 0: Transit signal priority not used (base case).

Case 1: Transit signal priority available in equal amounts (20-second green extensions) at all intersections along the arterial.

Case 2: Transit signal priority available in a limited fashion (10-second green extensions) at high volume cross street intersections with 20 -second green extensions available at all other intersections. 
Case 3: Transit signal priority unavailable at high volume cross street intersections, with 20-second green extensions available at all other intersections.

As with the previous analysis, far-side bus stop configurations and 10-minute bus headway were assumed. In addition, transit signal priority is only used by buses traveling in the peak period direction, the northbound direction.

The time frame used for this analysis began 6 minutes after the beginning of each TRAF-Netsim simulation and concluded 18 minutes after the beginning of each simulation. This analysis time frame was chosen because the northbound bus enters the network about 6 minutes after the beginning of each simulation and exits the network about 18 minutes after the beginning of each simulation.

To account for the variability that occurs over different simulation runs, each of the 4 cases was simulated twice. As a result, a total of eight simulations were run, with the results of two simulations averaged for each of the four cases.

The criterion that was used to compare the various transit signal priority strategies was the total travel time occurring along the cross street and arterial links during the analysis time frame.

Finally, the occupancy of the bus and autos was taken into consideration by multiplying the travel times (in vehicle minutes) by the occupancy rates (passengers per vehicle) to obtain the travel time along each link in terms of total person-minutes.

Table 7 shows the results of this analysis when bus occupancy is assumed to be 10 passengers per bus, accompanied by an average auto occupancy of 1.2 passengers.

As can be seen from Table 7, the base case outperformed all the cases where transit signal priority was used. As expected, the cross streets suffered with the use of transit signal priority, with the greatest increase in cross street travel time occurring with Case 1 . When priority is limited and restricted at the high volume cross street locations the travel times along the cross streets are reduced relative to Case 1, but are still greater than the base case.

The travel time that auto traffic experiences along the arterial fluctuates over the four cases with no apparent pattern. In addition, none of the changes are very 


\section{Table 7}

Total Travel Time (Person-Minutes) Within Arterial Network (Bus Occupancy $=10$, Auto Occupancy $=1.2$ )

\begin{tabular}{lrrrr}
\hline & Case 0 & Case 1 & Case 2 & Case 3 \\
\hline Auto travel time along arterial & 4405 & 4379 & 4376 & 4417 \\
Auto travel time along cross streets & 2899 & 3193 & 3023 & 2985 \\
Bus travel time along arterial & 108 & 89 & 96 & 99
\end{tabular}

Total Travel Time within Arterial Network7412 $\quad 7661 \quad 7494 \quad 7501$

Case 0 -Transit signal priority not used (base case).

Case 1-Transit signal priority available in equal amounts (20-second extensions) at all intersections.

Case 2-Limited transit signal priority available (10-second extensions) at high volume cross street intersections with 20 -second extensions available at all other intersections.

Case 3-Transit signal priority unavailable at high volume cross street intersections, with 20 -second green extenşions available at all other intersections.

large with respect to the base case. In fact, over the four cases, auto travel times along the arterial all fall within 1 percent of one another. This indicates that transit signal priority has little effect on the performance of automobile traffic traveling along the arterial which receives priority.

TRAF-Netsim's graphical animation showed that arterial traffic in the vicinity of the bus benefited from the bus's first priority call. However, after receiving priority, the bus typically stopped at a far-side bus stop, which caused it to lose coordination with the arterial traffic which benefited from the first priority call. As a result, the impact of signal priority upon arterial traffic may come in the form of increased number of stops and start up delay.

Finally, Table 7 shows that bus travel time is reduced relative to the base case over all three signal priority strategies, with the shortest bus travel time occurring with Case 1 . However, although bus travel time is reduced by significant percent- 
ages, the absolute travel time savings that the bus receives is minor compared with the absolute travel time increases imposed upon the cross streets when signal priority is initiated.

With 10-minute bus headways and significant automobile traffic volumes, bus mode share is extremely small. When bus occupancy is assumed to be 10 passengers, the bus's share of the total travel time within the network over the analysis period is only 1.5 percent (for the base case). Therefore, even improving the bus's performance significantly (on a percent basis) fails to provide the overall transportation network with significant absolute gains. In addition, the disruption caused to the network with the use of the signal priority timings overwhelms any small benefits realized by bus passengers.

Similar results were encountered when bus occupancy was assumed to be 25 and 50 passengers. Even with these occupancies, the bus's share of overall travel time was extremely small, causing the benefits of signal priority to be overshadowed by the resulting increased travel times along the cross streets.

This analysis leads to several conclusions. First, in areas where transit enjoys only a small mode share, transit signal priority is not recommended. However, in areas where transit enjoys a higher mode split, active signal priority may be feasible. In addition, the well-being of general traffic can be considered while using signal priority by compensating cross streets for lost green time or monitoring the saturation levels of cross streets. It is important to note, however, that if transit enjoys a high mode split within a particular transportation network, the network signal timings will almost certainly reflect the needs of the transit vehicles to begin with (perhaps in the form of passive priority). Therefore, under these circumstances active signal priority may not provide significant incremental transit benefits.

\section{Conclusions}

Reducing signal cycle lengths and split phasing are passive priority techniques that may be useful during off-peak times with local transit service. Reducing the cycle lengths along an arterial reduces transit delay and delay to general traffic if the arterial is operated with a generous amount of excess capacity. Split phasing, however, enjoyed only modest success. 
Unconditional signal priority during off-peak times offers express transit service significant potential benefits. However, its use should be regulated by placing limits on green extension and red truncation lengths, especially at intersections with busy cross streets.

During peak times, active transit signal priority should be used with caution. Active signal priority may cause disnuptions along highly saturated cross streets that do not dissipate before the next priority call. Far-side bus stops should be used with active signal priority to ensure that signal priority calls are not wasted as transit vehicles dwell at bus stops. Also, the success of transit signal priority during peak times is proportional to the transit mode share within the network. Only when transit gains a significant share of trips within the network will transit signal priority have an overall positive network impact. However, providing transit signal priority will improve transit service. Enhanced transit service promotes a more sustainable transportation mode within any transportation network and may, therefore, result in longterm benefits by encouraging transit use.

\section{References}

Al-Sahili, Khaled, and William C. Taylor. 1995. Bus preemption signal (BPS): An application, executive summary. Michigan State University.

Bowen, G. T., R. D. Bretherton, J.R. Landles, and D. J. Cook. 1994. Active bus priority in SCOOT. 7th International Conference on Road Traffic Monitoring and.

Hounsell, N. B., and J. P. Wu. 1995. Public transport priority in real-time traffic control systems. Applications of Advanced Technologies in Transportation Engineering.

Hounsell, N. B., F. N. McLeod R. D. Bretherton, and G. T. Bowen. 1996. PROMPT: Field trial and simulation results of bus priority in SCOOT. 8th International Conference on Road Traffic Monitoring and Control Priority in SCOOT.

Mainline Traffic Signal Priority Study. 1991. Phase 5-Demonstration project, final report. Municipality of Metropolitan.

Sunkari, Srinivasa R, Phillip S. Beasley, Thomas Urbanik II, and Daniel B. Fambro. 1995. Model to evaluate the impacts of bus priority on signalized intersections. Transportation Research Record 1494. Washington, DC: Transportation Research Board. 
TRAF User Reference Guide, Version 5.0.1995. U.S. Department of Transportation: Federal HighwayAdministration.

Urbanik II, Thomas, and R. W. Holder. 1977. Evaluation of priority techniques for high occupancy vehicles on arterial streets. College Station: Texas Transportation Institute, Texas A\&M University.

\section{About the Authors}

MichaEl GaRRow is an associate with Barton-Aschman Associates, Inc. specializing in transportation planning, traffic engineering, transit planning, and airport ground transportation planning.

RaNDY MACHEMEHL is a Professor and Associate Chairman of the Department of Civil Engineering at The University of Texas at Austin and serves as Associate Director of the Center for Transportation Research for the Southwest Region University Transportation Center. 